\title{
An infant with a palatal fistula secondary to Candida infection
}

\author{
Sarwpriya Sharma, \\ Jaideep Singh Chauhan \\ Department of Maxillofacial Surgery \\ and 'Smile Train' Cleft Centre, CHL \\ Hospitals, Indore, India
}

Candida osteomyelitis affecting maxillofacial bones has been scantly documented in the literature. Infantile osteomyelitis is an uncommon and life-threatening disease. Candida osteomyelitis causes significant morbidity. The present report describes a case of a 9-month-old infant with infantile osteomyelitis secondary to Candida infection. This report describes its presentation and the management of palatal fistula in an infant.

Keywords: Candida utilis / Infantile osteomyelitis / Osteomyelitis / Palatal fistula

\section{INTRODUCTION}

Candida osteomyelitis is a chronic form of invasive osteomyelitis. It causes significant morbidity if not recognized early or treated effectively [1,2] and may persist for months [3]. Depending on the mechanism of development, Candida osteomyelitis can be classified as (1) direct inoculation: seeding of bone tissue by external trauma, open wound, ulcer, or surgical manipulation; (2) contiguous infection: the presence of an infectious Candida process close to the subsequently infected bone; or (3) hematogenous infection: seeding of bone tissue by bloodstream route in the absence of contiguous or direct inoculation. Infantile osteomyelitis is an uncommon and life-threatening disease. Etiological considerations may include infective pathogenesis, genetic disorders, toxins, and/or environmental factors. Here, we report a rare case of a 9-month-old male infant diagnosed with infantile osteomyelitis secondary to Candida infection.

\section{CASE REPORT}

A 9-month-old male infant referred to our center with palatal

Correspondence: Sarwpriya Sharma

Department of Maxillofacial Surgery and 'Smile Train' Cleft Centre, CHL Hospitals,

AB Road, LIG Square, Indore 452008, India

E-mail: drsarwpriyasharma@gmail.com

Received April 7, 2020 / Revised May 14, 2020 / Accepted June 19, 2020 fistula secondary to a pathological condition. Antenatal history revealed a male neonate of $3.5 \mathrm{~kg}$ was delivered vaginally in the 39th week of gestation with no perinatal or postnatal complications. After standard postdelivery care, the baby was healthy at the time of discharge. The baby was admitted to the hospital when he was one and a half months old with a complaint of discharging sinus from the right cheek and whitish scrapable patches over his cheek and palate. Incision and drainage were performed extraorally, and culture swab was taken for examination. Bone biopsy was done from the palatal region, and the bone was exposed. Microscopic examination suggested areas of osteolysis, yeast-like fungus, and Candida utilis grew on cultures of the bone specimen. Culture swab reported the presence of a non-albican Candida, C. utilis. After the patient was treated with antifungal therapy (liposomal amphotericin B) for one and a half months, the baby improved clinically.

Then the patient presented to our center with a chief complaint of nasal regurgitation and feeding problems. Clinical examination identified an obvious deformity of the middle third of the face (Fig. 1). These findings were confirmed on a threedimensional reconstructive computed tomography, which also showed the destruction of maxilla, including a part of the nasal bone (Fig. 2). Intraoral examination revealed a large-sized, ovalshaped anterior palatal fistula with no inflammatory signs (Fig. 3). Deciduous teeth were absent with no maxillary alveolar 


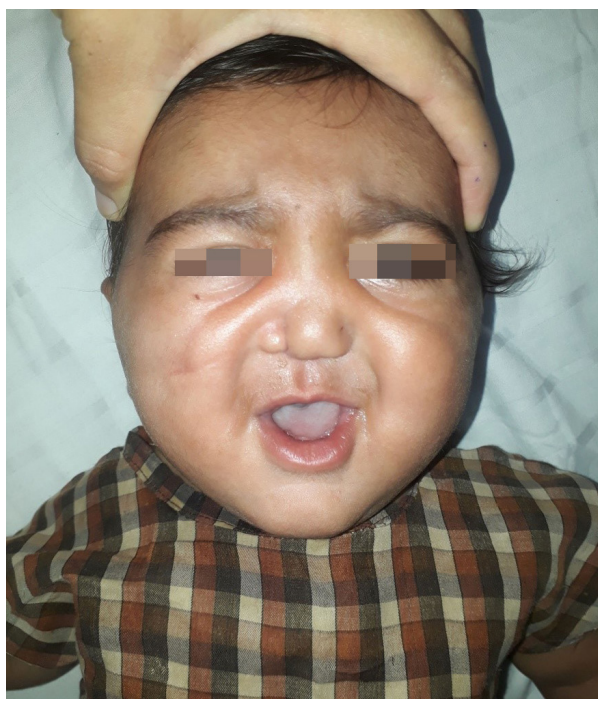

Fig. 1. A 9-month-old male infant showing facial deformity.

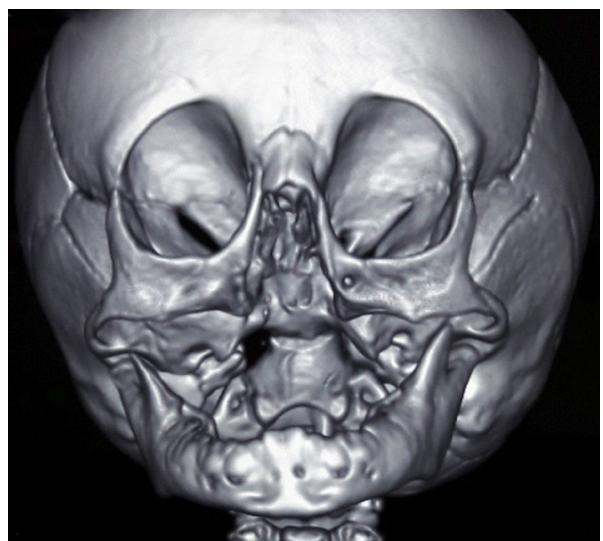

Fig. 2. Three-dimensional computed tomography scan.

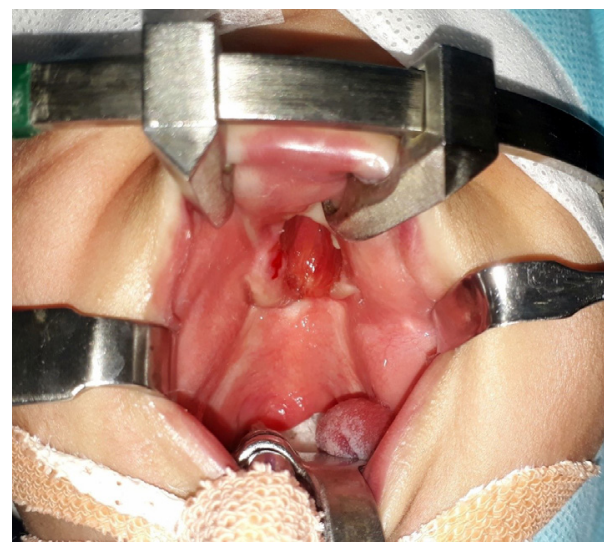

Fig. 3. Preoperative photograph showing palatal fistula.

arch. Depending on the history, culture report, and clinical and radiographical findings, a definitive diagnosis of Candida osteomyelitis was made. Maxillary reconstruction was not recommended as the patient's age was less than a year. We planned

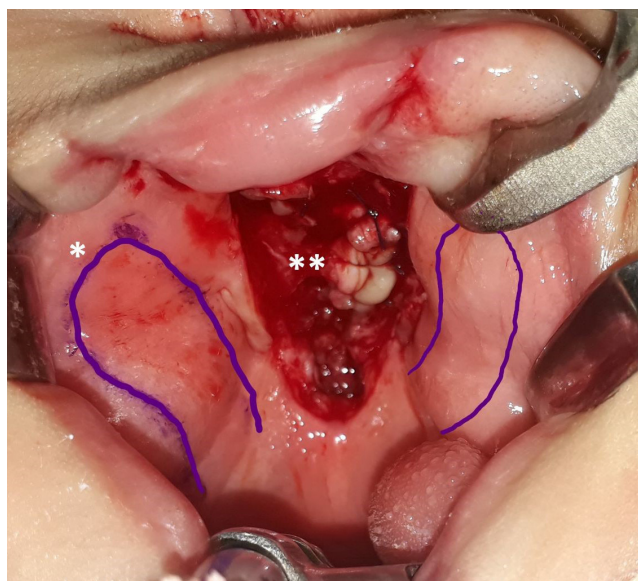

Fig. 4. Intraoperative photograph showing incision lines of posteriorly based myomucosal buccal flap (asterisk) and nasal lining closure (double asterisks).

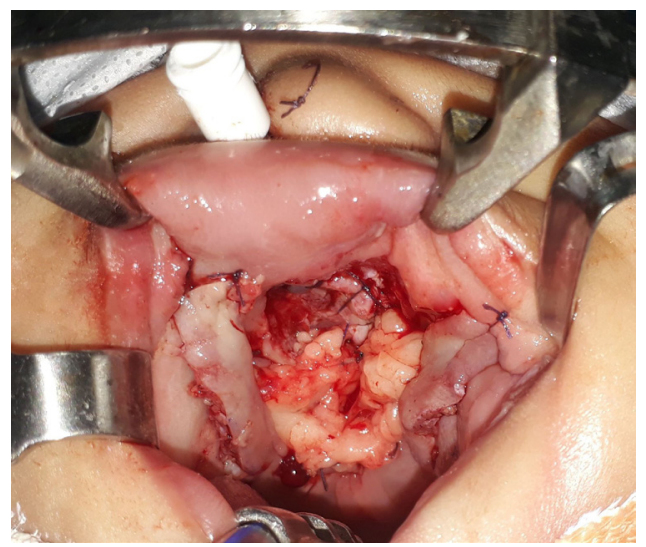

Fig. 5. Intraoperative photograph showing bilateral buccal flaps along with buccal fat pad.

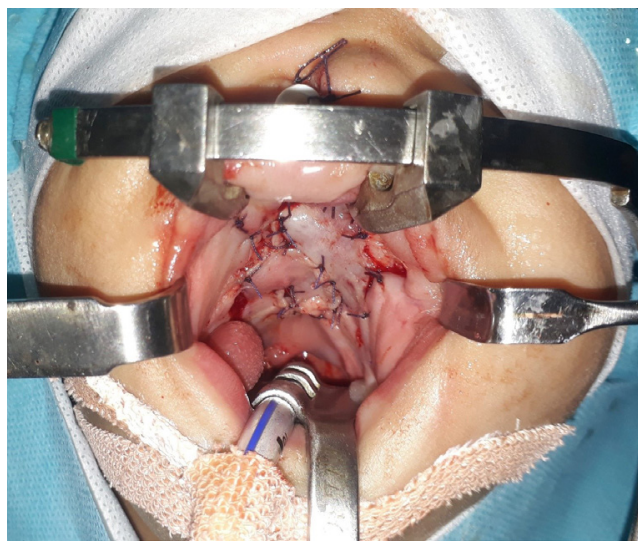

Fig. 6. Immediate postoperative photograph.

closure of the palatal fistula to overcome his present problems like feeding and nasal regurgitation. The patient was operated under general anesthesia. No signs of fibrosis were observed. The nasal layer was repaired, and the marking of posteriorly 


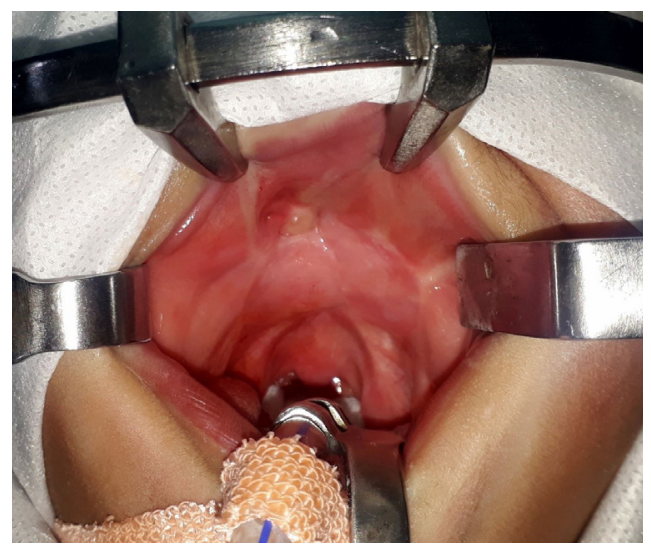

Fig. 7. Photograph 6 months after surgery.

based myo-mucosal buccal flaps was done. (Fig. 4). Bilateral buccal flaps were taken, along with a buccal fat pad, to cover the nasal layer and facilitate the closure (Figs. 5, 6). Complete closure was achieved (Fig. 7)

\section{DISCUSSION}

Candida osteomyelitis causes significant morbidity. Prior et al. [4] reported that C. utilis has long been known for its industrial applications, but it has rarely been described as an infectious agent in humans [5-7]. It is characterized by a chronic course with a male predominance. Hematogenous dissemination is the most common mechanism involved with this infection, followed by direct inoculation and contiguous infection. Based on age, the common sites for pediatric patients are the femur, humerus, and vertebra/ribs. The costal cartilage, costochondral joints, knees, and sacroiliac joint are the other usual sites. Maxillofacial bones are less commonly affected by Candida osteomyelitis. Gamaletsou et al. [8] conducted a study in 2012 and analyzed 207 pediatric and adult cases (1970-2011) of Candida osteomyelitis and concluded that timely diagnosis of Candida osteomyelitis with extended courses of 6-12 months of antifungal therapy and surgical intervention may improve the outcome of the disease. The study also documented the importance of relapse despite treatment, possibly as a result of inadequate duration of therapy.

Risk factors for candidemia in neonates and infants include umbilical vein catheterization, very low birth weight, necrotizing enterocolitis, localizing osteoarticular symptoms, etc. In our case, there was no such history and the baby's weight was 3.5 $\mathrm{kg}$. Predisposing factors also include the usage of antineoplastic agents and the immunocompromised status of the patient. Compromised immunity might be the causative factor in our patient.
Gathe et al. [1] reported five cases of Candida osteomyelitis and employed successful therapeutic regime. They suggested the combination of antifungal therapy (most often amphotericin B) with surgical debridement. They also proposed that amphotericin B should be considered as the drug of choice until controlled studies demonstrate the superiority of the other agents in deep-seated Candida infection. Andermahr et al. in 1988 [9] reported a case of Candida tropicalis spondylitis L I and L II with Candida coxitis and used the combination of antifungal therapy (amphotericin B or fluconazole) with radical surgical debridement for the treatment. Similarly, in our case, liposomal amphotericin B was prescribed for one and a half months parenterally. Arranz-Caso et al. in 1996 [10] reported a case of zygomatic candidiasis osteomyelitis in an adult patient with history of diabetes and oral candidiasis. He reported that fluconazole was ineffective, and amphotericin B was proven to be successful in the treatment.

In our case, Candida osteomyelitis affects complete maxillary bone and the aggressiveness of the infection was so destructive that it involved the maxillary alveolar process along with the basal bone and a part of nasal bone in the acute course of duration.

To conclude, Candida osteomyelitis affecting maxillofacial bones is a rare entity, and very few cases have been reported in the literature. Early diagnosis and prompt treatment can reduce the incidence of the sequel.

\section{NOTES}

\section{Conflict of interest}

No potential conflict of interest relevant to this article was reported.

\section{Ethical approval}

The study was approved by the Integrity Ethical Committee of CHL Hospitals (approval No. CHL/DEN/JSC/JUN-2019/07) and performed in accordance with the principles of the Declaration of Helsinki. Written informed consent was obtained.

\section{Patient consent}

The patient's parent provided written informed consent for the publication and the use of his images.

\section{ORCID}

Sarwpriya Sharma https://orcid.org/0000-0001-9565-3490 Jaideep Singh Chauhan https://orcid.org/0000-0002-1558-6505 


\section{REFERENCES}

1. Gathe JC Jr, Harris RL, Garland B, Bradshaw MW, Williams TW Jr. Candida osteomyelitis: report of five cases and review of the literature. Am J Med 1987;82:927-37.

2. Collignon P. Candida osteomyelitis. Am J Med 1987;83:1173.

3. Bellini C, Antonini P, Ermanni S, Dolina M, Passega E, Bernasconi E. Malignant otitis externa due to Aspergillus niger. Scand J Infect Dis 2003;35:284-8.

4. Prior B, Kilian S, Lategan P. Growth of Candida utilis on ethanol and isopropanol. Arch Microbiol 1980;125:133-6.

5. Alsina A, Mason M, Uphoff RA, Riggsby WS, Becker JM, Murphy D. Catheter-associated Candida utilis fungemia in a patient with acquired immunodeficiency syndrome: species verification with a molecular probe. J Clin Microbiol 1988;26: 621-4.

6. Hazen KC, Theisz GW, Howell SA. Chronic urinary tract in- fection due to Candida utilis. J Clin Microbiol 1999;37:824-7.

7. Shih MH, Sheu MM, Chen HY, Lin SR. Fungal keratitis caused by Candida utilis: case report. Kaohsiung J Med Sci 1999;15: 171-4.

8. Gamaletsou MN, Kontoyiannis DP, Sipsas NV, Moriyama B, Alexander E, Roilides E, et al. Candida osteomyelitis: analysis of 207 pediatric and adult cases (1970-2011). Clin Infect Dis 2012;55:1338-51.

9. Andermahr J, Isenberg J, Prokop A, Rehm KE. Candida spondylitis: case report and review of the literature. Unfallchirurg 1998;101:955-9.

10. Arranz-Caso JA, Lopez-Pizarro VM, Gomez-Herruz P, GarciaAltozano J, Martinez-Martinez J. Candida albicans osteomyelitis of the zygomatic bone: a distinctive case with a possible peculiar mechanism of infection and therapeutic failure with fluconazole. Diagn Microbiol Infect Dis 1996;24:161-4. 